\title{
Estudio comparativo de mujeres mayores de noventa años y ancianas menores institucionalizadas
}

\author{
Pedro Paulo Marín L, Homero G ac E, Trinidad Hoyl M, \\ Marcela Carrasco G, Patricio Duery Da, \\ Mauricio Cabezas U ${ }^{a}$, Kristina Petersen $C^{a}$, \\ Catalina D ussaillant $K^{b}$, Eduardo Valenzuela $A$.
}

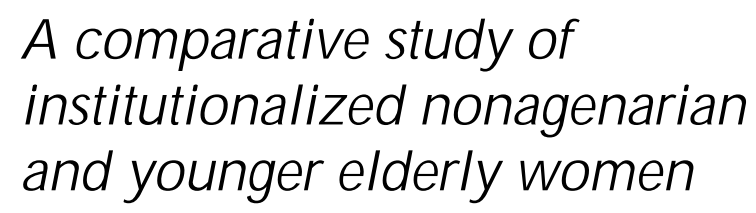

Background: The number of nonagenarians is rapidly growing in Chile. This age group is mainly female, with higher frailty markers and in higher risk of being placed at nursing homes. Aim: To describe features of nonagenarian women and compare them with a group of women between 60-89 years, both living in nursing homes, in terms of disability and resource use at the institution. Subjects and methods: A total of 230 nonagenarian women and 460 women, aged 60-89 years, were evaluated in the nursing home Fundación Las Rosas de Ayuda Fraterna (only for poor elderly) in Santiago, Chile. The assessment instruments were the Geriatric Assessment instrument FEGAUC, functional and mental evaluation scales of Spanish Red Cross and the Resource Utilization System, RUG T18, an independent diagnostic classification system that allows the determination of resource use in terms of cost and personnel needs. Results: Nonagenarian women had significantly $(p<0.05)$ more disability (falls, urinary incontinence, memory problems and mobility difficulties), and were classified in RUG categories of higher resource utilization and dependency than younger women. Nevertheless, nonagenarians were a very heterogeneous group, almost half of them were able of moving by themselves or required little assistance and had minimal memory problems. Conclusions: Nonagenarian women assessed in this institution are an heterogeneous group, some with minimal disability and other more frail and dependent than the younger elderly women, being classified in higher categories of resource utilization RUG T18 (Rev Méd Chile 2004; 132: 33-9).

(Key Words: Aged, 80 and over; Frail elderly; Geriatric assessment; Geriatric nursing)

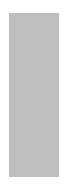

Recibido el 13 de mayo, 2003. Aceptado en versión corregida el 21 de octubre, 2003.

Programa de Geriatría y Gerontología, Departamento de Medicina Interna, Facultad de Medicina, Pontificia Universidad Católica de Chile. Santiago, Chile.

anternos de Medicina, Facultad de Medicina Pontificia Universidad Católica.

bAlumna de Medicina, Facultad de Medicina Pontificia Universidad Católica.

Correspondencia a: Dr. Pedro Paulo Marín L Programa de

Geriatría y Gerontología, Departamento de Medicina Interna,

Facultad de Medicina, Pontificia Universidad Católica de Chi-

le. Casilla 114-D, Santiago Chile. E mail: ppmarin@med.puc.cl 
$E^{s}$ un hecho conocido que la población mundial está envejeciendo, pero esta realidad es particularmente importante en países en vías de desarrollo como Chile, por la rapidez con que está ocumiendo, sus consecuencias en salud pública y los desafíos que plantea. Los Adultos Mayores de sesenta años (AM), según datos del CENSO 2002, aumentaron en la última década del $9,8 \%$ de la población total al $11,4 \%$, equivalente a 1.717 .478 personas actualmente. En términos de crecimiento, mientras que la población general creció a una tasa de 1,2\% anual, los AM lo hicieron a un ritmo de 3,3\% y dentro de este grupo el que más se incrementó fue el de los mayores de 90 años, que aumentó de 25.682 a 38.556 personas, correspondiendo al 0,26\% de la población total del país, observándose un envejecimiento del grupo de $\mathrm{AM}^{1}$.

Los nonagenarios son un grupo aún reducido, pero en expansión, aumentando así su importancia relativa y planteando un importante desafío de salud pública para nuestro país. Ellos tienen varias particularidades que hay que considerar para planificar futuras estrategias de atención. Por ejemplo, hay una clara predominancia del género femenino $(68,1 \%)^{1}$, debido a la mayor expectativa de vida de las mujeres; además, en este segmento de edad se concentran los más frágiles y dependientes, lo que se correlaciona con un mayor riesgo de ser institucionalizado y de mortalidad, acarreando mayores costos de atención ${ }^{2-5}$.

A pesar de lo anterior, es muy escasa la información disponible de las características de este grupo en Chile $^{3}$ y menos aún de los nonagenarios institucionalizados que representan un grupo especialmente vulnerable.

El objetivo del presente trabajo es realizar un estudio transversal descriptivo de las características de mujeres institucionalizadas pobres de 90 años o más, en una zona urbana, analizando el nivel de fragilidad, discapacidad y recursos de atención que reciben, comparándolas con un grupo de mujeres institucionalizadas pero más jóvenes.

\section{PACIENTES Y MÉTOdos}

El presente trabajo descriptivo, se realizó durante el mes de diciembre de 2002, en Santiago, en los hogares de institucionalización de AM de la
Fundación Las Rosas de Ayuda Fraterna. Se evaluó a todas las mujeres nonagenarias residentes $(n=$ 230) y se comparó con un grupo aleatorio de 460 mujeres entre 60 y 89 años, de la misma institución.

La evaluación consistió en la aplicación de tres instrumentos: Ficha de Evaluación Geriátrica Ambulatoria FEGAUC $^{6}$, cuestionario de tamizaje de los principales factores de riesgo de fragilidad del AM validado previamente en pacientes ambulatorios; Escalas de Valoración Geriátrica de la Cruz Roja Española ${ }^{7,8}$ que clasifica, por medio de una puntuación simple de 0 a 5 la situación mental y funcional, siendo la puntuación mayor la de mayor discapacidad; y por último, Sistema de Clasificación de pacientes (case mix) Resources Utilization Groups» en su versión RUG T-18, que es una clasificación independiente del diagnóstico del sujeto y permite inferir el nivel de utilización de recursos, tanto en necesidad de personal como en costo de atención del paciente; se usó ya que es sencilla de completar y nuestro grupo tiene experiencia en su uso ${ }^{9}$. El sistema de clasificación RUG T-18 agrupa a cada paciente en un determinado grupo, basándose en sus «Características Clínicas»que son las condiciones que llevan a clasificarle jerárquicamente en los grupos de rehabilitación, cuidados especiales clínicamente complejos, trastornos de conducta, funciones físicas reducidas y posteriormente se subclasifican dentro de cada grupo en función del Indice de Actividades de la Vida Diaria (AVD).

La aplicación de los instrumentos fue llevada a cabo por miembros del Equipo de Geriatría, internos y alumnos de la Facultad de Medicina de la Pontificia Universidad Católica de Chile y personal del equipo de salud de la Fundación Las Rosas de Ayuda Fraterna, todos previamente capacitados.

Para el análisis estadístico se utilizó el programa SPSS, se realizó comparación de promedios con el test t de Student y se comparó porcentajes con el test de Chi cuadrado. Se consideró significativo un valor $\mathrm{p}<0,05$.

\section{Resultados}

El grupo de las nonagenarias $(n=230)$ tiene un promedio de edad de 92,7 años \pm 4,7 DE (90 a 106 
años) versus el grupo de mujeres institucionalizadas jóvenes con un promedio de edad de 82,4 años $\pm 4,8 \mathrm{DE}$ ( 60 a 89 años).

En la Tabla 1 se muestran los resultados encontrados al aplicar la Ficha de Evaluación Geriátrica Ambulatoria FEGAUC, observándose que independiente de la edad casi el $20 \%$ de estas mujeres está recibiendo cinco o más fármacos, el $44 \%$ no tiene hijos y el $56 \%$ de ellas refiere sentirse deprimidas. El grupo de las nonagenarias refiere significativamente $(p<0,05)$ más problemas con su memoria ( 72 vs $57 \%$ ), un porcentaje mayor de incontinencia urinaria (48 vs 38\%), más caídas en el último año (35 vs $27 \%$ ) y en general mayores limitaciones en su movilidad, en comparación con el grupo de mujeres más jóvenes.

En la Tabla 2 se presentan los resultados de las Escalas de la Cruz Roja Española, observándose

Tabla 1. Resultados encontrados en mujeres institucionalizadas con la «Ficha FEG AU C», analizados según edad

\begin{tabular}{|lccr|}
\hline & $\begin{array}{c}60-89 \text { años } \\
\mathrm{n}=460\end{array}$ & $\begin{array}{c}\geq 90 \text { años } \\
\mathrm{n}=230\end{array}$ & $\mathrm{p}$ \\
\hline Toma más de 5 fármacos & $19,6 \%$ & $17 \%$ & 0,407 \\
Sin hijos & $42,7 \%$ & $46,1 \%$ & 0,398 \\
Se ha caído en el último año & $27,2 \%$ & $34,8 \%$ & 0,039 \\
Refiere problemas de memoria & $57,2 \%$ & $71,6 \%$ & $<0,001$ \\
Incontinencia urinaria & $37,6 \%$ & $47,8 \%$ & 0,010 \\
Clínicamente enflaquecido & $20,4 \%$ & $33,5 \%$ & $<0,001$ \\
No recuerda tres palabras & $68,7 \%$ & $82,1 \%$ & $<0,001$ \\
Refiere sentirse deprimido & $55 \%$ & $58,1 \%$ & 0,460 \\
No puede vestirse solo & $41,7 \%$ & $58,3 \%$ & $<0,001$ \\
No se para de la silla & $29,5 \%$ & $42,6 \%$ & 0,001 \\
No camina 5 pasos & $31,1 \%$ & $44,8 \%$ & $<0,001$ \\
No se levanta de la cama & $30,3 \%$ & $43,9 \%$ & $<0,001$ \\
\hline
\end{tabular}

Tabla 2. Resultados obtenidos en mujeres institucionalizadas al aplicar las «Escalas de la C ruz Roja Española», analizados según edad

\begin{tabular}{|lccrr|}
\hline & Total & $60-89$ años & $\geq 90$ años & \\
& $n=690$ & $n=460$ & $n=230$ & $p$ \\
\hline Evaluación Funcional & & & & \\
0= Camina normal, se vale por sí mismo & $29,4 \%$ & $35,4 \%$ & $17,4 \%$ & $<0,001$ \\
1= Algunas dificultades desplazamientos complejos & $17,5 \%$ & $18 \%$ & $16,5 \%$ & 0,620 \\
2= Ocasional apoyo con bastón o similar & $24,2 \%$ & $22,2 \%$ & $28,3 \%$ & 0,078 \\
3= Graves dificultades AVD; ayuda de 1 persona & $8,0 \%$ & $6,7 \%$ & $10,4 \%$ & 0,001 \\
4= Ayuda AVD de 2 personas & $7,7 \%$ & $7 \%$ & $9,1 \%$ & 0,312 \\
5= Inmovilizado, cuidados continuos, incontinente & $13,2 \%$ & $10,7 \%$ & $18,3 \%$ & 0,005 \\
& & & & \\
Evaluación Mental & & & & \\
0= Normal & $34,2 \%$ & $39,8 \%$ & $23 \%$ & $<0,001$ \\
1= Trastornos leves de memoria; conversación normal & $22,5 \%$ & $22,6 \%$ & $22,2 \%$ & 0,897 \\
2= Clara alteración memoria; conversación imperfecta & $18,1 \%$ & $15,9 \%$ & $22,6 \%$ & 0,030 \\
3= Severas alteraciones en la memoria y/u orientación & $9,7 \%$ & $6,7 \%$ & $15,7 \%$ & $<0,001$ \\
4= Desorientación completa & $8,6 \%$ & $9,1 \%$ & $7,4 \%$ & 0,441 \\
5= Demencia avanzada vida vegetativa & $7,0 \%$ & $5,9 \%$ & $9,1 \%$ & 0,112 \\
\hline
\end{tabular}


que en general las nonagenarias están en peores condiciones en el aspecto funcional que el grupo entre 60 y 89 años. Así como es significativamente menor el porcentaje de nonagenarias que camina normal o se vale por sí misma $(17,4 \%$ vs $35,4 \%)$ y son mayores los porcentajes de aquellas que tienen graves dificultades en ABVD o requieren ayuda de una persona para movilizarse $(10,4 \%$ vs $6,7 \%)$, se encuentran totalmente inmovilizadas 0 incontinentes (18,3\% vs $10,7 \%)$. Con respecto a la evaluación mental, observamos algo muy similar en las nonagenarias, siendo significativamente menor el porcentaje evaluado como normal (23\% vs $39,8 \%$ ) y significativamente mayor el de las que tienen claras o severas alteraciones en la memoria u orientación $(15,7$ vs $6,7 \%)$. En cambio, no observamos diferencias entre ambos grupos respecto al porcentaje de aquellos catalogados como portadores de demencia avanzada.
En la Tabla 3 se presentan los resultados encontrados al aplicar el «istema de clasificación de pacientes RUG-T18». Destaca que las mujeres más jóvenes, presentan estadísticamente menor frecuencia de incontinencia urinaria $(34,6 \%$ vs $45,7 \%)$ y menos problemas con su movilidad, en cambio, ambos grupos son similares con respecto a la capacidad de alimentarse por sí mismo (82,4\% vs $78,7 \%$ ) y en ambos grupos menos del $20 \%$ es completamente dependiente en su alimentación. Los datos previos explican por qué el índice de AVD sea mayor (peor) en las nonagenarias. Al comparar la clasificación de las «categorías jerárquicas del RUG-T18», encontramos que menos del $10 \%$ (categorías $\mathrm{S}+\mathrm{C}$ ) son portadores de cuadros más agudos o de mayor utilización de recursos y no hay pacientes que cumplan con los criterios para decir que reciben rehabilitación (recibir al menos media hora de terapia diaria). Sin embargo,

\section{Tabla 3. Resultados obtenidos en mujeres institucionalizadas con el Sistema de clasificación de pacientes -RU G -T 18}

\begin{tabular}{|c|c|c|c|c|}
\hline Actividades de la vida diaria (AVD) & Total & $\begin{array}{c}60-89 \text { años } \\
n=460\end{array}$ & $\begin{array}{c}\geq 90 \text { años } \\
n=230\end{array}$ & $\mathrm{p}$ \\
\hline \multicolumn{5}{|l|}{ Continencia } \\
\hline Autosuficiente & $60,1 \%$ & $63,7 \%$ & $53,0 \%$ & 0,007 \\
\hline Requiere ayuda & $38,3 \%$ & $34,6 \%$ & $45,7 \%$ & 0,004 \\
\hline En reentrenamiento vesical & $1,6 \%$ & $1,7 \%$ & $1,2 \%$ & 0,667 \\
\hline \multicolumn{5}{|l|}{ Alimentación } \\
\hline Autosuficiente & $81,2 \%$ & $82,4 \%$ & $78,7 \%$ & 0,241 \\
\hline Requiere ayuda continuamente & $5,9 \%$ & $5,9 \%$ & $6,1 \%$ & 0,909 \\
\hline Completamente dependiente & $12,6 \%$ & $11,3 \%$ & $15,2 \%$ & 0,144 \\
\hline Con SNG-Parenteral & $0,3 \%$ & $0,4 \%$ & $0 \%$ & 0,316 \\
\hline \multicolumn{5}{|l|}{ Movilidad } \\
\hline Independiente & $57,4 \%$ & $61,7 \%$ & $48,7 \%$ & 0,001 \\
\hline Ayuda de 1 persona & $22,9 \%$ & $21,3 \%$ & $26,1 \%$ & 0,158 \\
\hline Ayuda de 20 más personas & $19,7 \%$ & $17,0 \%$ & $25,2 \%$ & 0,010 \\
\hline Indice AVD (promedio \pm DS) & $4,36 \pm 1,70$ & $4,23 \pm 1,67$ & $4,62 \pm 1,75$ & \\
\hline \multicolumn{5}{|l|}{ Categorías jerárquicas } \\
\hline $\mathrm{R}=$ Rehabilitación & $0 \%$ & $0 \%$ & $0 \%$ & - \\
\hline $\mathrm{S}=$ Cuidados especiales & $1 \%$ & $1,1 \%$ & $0,9 \%$ & 0,788 \\
\hline $\mathrm{C}=$ Clínicamente complejos & $8,6 \%$ & $8,3 \%$ & $9,1 \%$ & 0,700 \\
\hline $\mathrm{B}=$ Alteraciones de la conducta & $21,3 \%$ & $15 \%$ & $33,9 \%$ & $<0,001$ \\
\hline $\mathrm{P}=$ Institucionalizado & $69,1 \%$ & $75,7 \%$ & $56,1 \%$ & $<0,001$ \\
\hline
\end{tabular}


aun cuando tanto las nonagenarias como las mujeres de 60-89 años se agrupan preferentemente en la categoría de menor complejidad ( $\mathrm{P}$ : Institucionalizado) con un 56 y $76 \%$ respectivamente del total de cada grupo, hay diferencias estadísticamente significativas ya que el grupo de nonagenarias es más importante que el de las jóvenes en la categoría de alteraciones de la conducta $(33,9 \%$ vs $15 \%)$ y observamos que en general las mujeres más jóvenes supera a las nonagenarias en la categoría más simple (institucionalizado), lo que implica que requieren menos personal de salud en su cuidado y que, por lo tanto, el costo final del cuidado debe ser menor que las nonagenarias.

\section{DisCUSIÓN}

Así como el siglo XX fue el siglo del crecimiento demográfico, el siglo XXI será el del envejecimiento poblacional. Este fenómeno epidemiológico que era propio de países desarrollados, hoy es una realidad en países en vías de desarrollo como Chile, en que el grupo de AM actualmente alcanza el $11,4 \%$ del total de la población y es el segmento que más creció en la última década, destacando especialmente el grupo de los nonagenarios que aumentaron de 25.682 a 38.556 personas ${ }^{1}$.

El fenómeno del envejecimiento poblacional plantea grandes desafíos económicos, sociales y de salud pública, que deben ser enfrentados con una visión de optimización de recursos, sin embargo toda planificación en salud, requiere conocer las características del grupo en cuestión y considerar siempre sus variantes respecto a otros grupos de edad. Se sabe que la población anciana es heterogénea, ya que incluye, tanto a personas que aún son independientes, activas y en buenas condiciones de salud, como a otros -generalmente los más ancianos- que a menudo presentan mayor dependencia o discapacidad incluso requiriendo cuidados institucionalizados. En nuestro país se desconoce el número y las características de los AM nonagenarios institucionalizados ${ }^{2}$. La única publicación disponible, que es de nuestro grupo, reveló que los parámetros de salud no fueron significativamente peores en un grupo de nonagenarios que vivían en la comunidad comparados con los institucionalizados, teniendo estos últimos una red social más escasa ${ }^{3}$.

Considerando lo anterior, decidimos realizar este estudio descriptivo, utilizando como instrumentos de evaluación el sistema de clasificación de pacientes «Resource Utilization Group T-18» (RUG T-18) ${ }^{9}$, las escalas geriátricas de las Cruz Roja $^{6,8}$ y la escala FEGAUC 6 , todos métodos validados en la literatura.

Los resultados de este estudio, usando los tres instrumentos de valoración, muestran que las mujeres nonagenarias pobres e institucionalizadas en comparación con las mujeres de 60-89 años presentan significativamente mayor limitación funcional, fundamentalmente en cuanto a su movilidad, medido en parámetros como su capacidad de caminar sin ayuda, pararse de una silla, caminar cinco pasos o levantarse de la cama y más caídas en el último año. Esto representa y se relaciona con mayor fragilidad, discapacidad, mayor riesgo de institucionalización, mortalidad y finalmente mayor utilización de recursos tanto humanos como económicos. Estos resultados concuerdan con reportes bibliográficos previos como lo encontrado en una cohorte danesa de 2.262 nonagenarios de la comunidad $2,4,5,9,10$.

Otro hallazgo significativo es que un mayor número de mujeres nonagenarias son clasificadas como «clínicamente enflaquecidas». Aun cuando esto es una apreciación subjetiva, es un llamado de alerta sobre la necesidad de realizar una evaluación nutricional objetiva para detectar personas en riesgo nutricional o desnutridas y adecuar los aportes alimentarios que reciben estas mujeres. Existe una correlación interesante entre el estado nutricional, la sarcopenia y la inmovilidad, ya que se potencian ${ }^{11}$.

En cuanto a la evaluación mental el grupo de nonagenarias también presenta peores parámetros, en comparación con el grupo de mujeres institucionalizadas de menor edad, concordando los tres instrumentos de valoración, cada uno en su enfoque. Aun cuando un $45 \%$ de las mayores de 90 años queda clasificada en la escala de la Cruz Roja como normal o con trastornos leves de memoria, en el análisis comparativo por edad las mujeres de 60-89 años son significativamente más en la categoría normal y menos en las categorías 2 
y 3, (alteraciones claras y severas de memoria), aun cuando en la categoría de demencia avanzada no alcanzó diferencias estadísticamente significativas. En FEGAUC también es significativamente mayor el porcentaje de nonagenarias que fallan en el test de tres palabras y por último en el RUG quedan más nonagenarias en la categoría de alteraciones de conducta. Esto es importante ya que el deterioro cognitivo es un factor asociado a mayor mortalidad en pacientes ancianos ${ }^{5,12}$.

En cuanto a la continencia vemos que es claramente diferente la valoración entre los grupos, con mayor presencia de incontinencia urinaria en las nonagenarias. Esto se observa en la evaluación de continencia de RUG y en FEGAUC. Probablemente este hallazgo, se relaciona con los aspectos de inmovilidad y trastorno cognitivo antes expuesto.

Respecto al consumo de fármacos el 18,7\% de las pacientes estudiadas consume más de 5 medicamentos, no presentándose diferencias estadísticamente significativas respecto a la edad. Esta cifra es muy superior al $4 \%$ descrito en AM ambulatorios $^{7}$ y podría relacionarse con la alta prevalencia de demencia en esta población y el control de problemas conductuales. Es importante recalcar que la polifarmacia, que considera tanto los automedicados como los recetados por médico, es un factor de riesgo de desarrollar reacciones adversas a medicamentos ${ }^{13} \mathrm{y}$ aumenta el riesgo de caídas en el AM contribuyendo a la condición de fragilidad de estas mujeres. Las caídas pueden deteriorar la funcionalidad del AM y ser un gatillante de institucionalización o muerte ${ }^{10,14}$. Esto plantea un nuevo desafío de intervención futura en este grupo especialmente vulnerable.

Otro hallazgo relevante, que confirma lo descrito en la literatura chilena ${ }^{3}$ es el gran número de nonagenarias institucionalizadas sin hijos, independiente de la edad. Este hecho es importante ya que podría ser un factor determinante de una mala red de apoyo social y otro gatillante de institucionalización en adultos mayores nonagenarios.

Encontramos, entonces que las nonagenarias institucionalizadas incluidas en este estudio están en una situación de mayor fragilidad que el grupo comparativo más joven, siendo considerables sus limitaciones en las actividades básicas de la vida diaria. En varios estudios, sin embargo, se observa que la edad no se relaciona con la mortalidad final derivada de algún evento o procedimiento como ha sido descrito al estudiar la sobrevida luego de la implantación de marcapaso ${ }^{19}$ o para cirugía cardíaca $^{20}$. Lo mismo se aprecia al alta de los intensivos quirúrgicos donde influye fundamentalmente la severidad de las enfermedades de base 0 la cirugía en sí misma ${ }^{18,19}$. Por lo tanto, esto apoya la importancia de optimizar la calidad de atención de los AM nonagenarios, ya que la edad por sí sola no los excluye de los tratamientos habituales.

Por otra parte, un análisis optimista podría hacernos ver que, contrariamente a lo que la opinión pública piensa, casi la mitad de las nonagenarias son cognitivamente normales o tienen un trastorno de memoria leve que les permite tener conversaciones sin inconvenientes, casi la mitad no requiere ayuda de terceros para su desplazamiento (a lo más uso de bastón), no se sienten más deprimidas que otros adultos mayores y por último más de $50 \%$ de ellas son autosuficientes en su continencia y casi el $80 \%$ en su alimentación. Lo que demuestra la gran heterogeneidad de este grupo y concuerda con datos nacionales e internacionales de nonagenarios no institucionalizados ${ }^{3-5}$.

En conclusión, la población estudiada es más frágil que los adultos mayores más jóvenes, pero su situación es mejor de lo que a priori pudiera pensarse y por tanto se hace necesario un enfrentamiento activo del equipo de salud, con un enfoque individualizado, multidisciplinario y global que intervenga en la prevención, manejo y rehabilitación de la discapacidad, con el fin de mejorar la calidad de vida de estos adultos mayores. Estudios como éste son fundamentales para una adecuada planificación del tipo de servicios que los $\mathrm{AM}$ van a requerir en Chile $\mathrm{y}$ para que las instituciones puedan comparar el tipo de adultos mayores que atienden. 


\section{REFERENCIAS}

1. Instituto Nacional de Estadística (INE). Censo de población y vivienda, Chile. Resultados Generales. 2002.

2. MARín PP. Demografía y epidemiología del envejecimiento en Chile. En: Marín PP, Manual de Geriatría y Gerontología. Santiago: Ediciones Universidad Católica de Chile, 2002; 47-54.

3. Marín PP, Castro S, Gaieb I, Valenzuela E. Estudio comparativo de nonagenarios que viven en sus propios hogares versus los institucionalizados. Rev Méd Chile 1998; 126: 761-8.

4. Nybo H, Gaist D, Jeune B, McGue M. Functional status and self-rated health in 2,262 nonagenarians: the Danish 1905 Cohort Survey. J Am Geriatr Soc 2001; 49: 601-9.

5. Nybo H, Petersen HC, Gaist D, Jeune B. Predictors of Mortality in 2,249 Nonagerians. The Danish 1905, Cohort Survey. J Am Geriatr Soc 2003; 51: 1365-73.

6. Marín PP, Valenzuela E, Saito N, Castro S. Experiencia piloto en el uso de una ficha de evaluación geriátrica ambulatoria. Rev Méd Chile 1996; 124: 701-6.

7. Marín PP, Kornfeld R, Somlai E, Valenzuela E. Valoración geriátrica ambulatoria de 2,116 adultos mayores pobres. Rev Méd Chile 1998; 126: 609-14.

8. González Ji, Rodríguez M, Diestro P, Casado MT. Valoración funcional: relación de la escala de la Cruz Roja con el índice de Katz. Rev Esp Geriatr Gerontol 1991; 26: 197-202.

9. Marín PP, Valenzueia E, Castro S, Rodríguez S. Resultados al aplicar el sistema de clasificación de pacientes «RUG-T18»en ancianos ingresados a un hospital universitario. Rev Méd Chile 1998; 126: 761-8.

10. TinetTi ME. Preventing falls in elderly persons. $\mathrm{N}$ Engl J Med 2003; 348: 42-9.
11. GREENLUND LJ, NAIR KS. Sarcopenia-consequences, mechanisms and potential therapies. Mech Ageing Dev 2003; 124: 287-99.

12. Andersen K, Nybo H, Gaist D, Petersen HC. Cognitive impairment and mortality among nonagenarian: The Danish 1905 cohort survey. Dement Geriatr Cogn Disord 2002; 13: 156-63.

13. González G, Marín PP, Pereira G. Características de las caídas en el adulto mayor que vive en la comunidad. Rev Méd Chile 2001; 129: 1021-30.

14. TinetTi ME, Wiшams CS. Falls, Injuries due to falls and the risk of admission to a nursing home. N Engl J Med 1997; 337: 1279-84.

15. Margulies DR, LekAwa ME, BJerke HS, Hiatt JR. Surgical intensive care in the nonagenarian. No basis for age discrimination. Arch Surg 1993; 128 : 753-6; discussion 756-8.

16. Rigberg D, Cole M, Hiyama D, McFadden D. Surgery in the nineties. Am Surg 2000; 66: 813-81.

17. Von Strauss E, Fratigloni L, Vittanen M, Forseil Y. Morbidity and comorbidity in relation to functional status: a community based study of the oldest old (90+ years). J Am Geriatr Soc 2000; 48: 1462-9.

18. González-Martin G, Yáñez L, Valenzuela E et al. Reacciones adversas a medicamentos en pacientes geriátricos hospitalizados. Estudio prospectivo. Rev Méd Chile 1997; 125: 1129-36.

19. Shen WK, Hayes DL, Hammll SC, Bailey KR. Survival and functional independence after implantation of a permanent pacemaker in octogenarians and nonagenarians. A population based study. Ann Intern Med 1996; 125: 476-80.

20. Vermeulen T, Rodrigus I, Stockman B, Amsel B, Mouljn A. Cardiac surgery in octogenarians. Acta Cardiol 2001; 56: 367-73.

Agradecimientos:

Se agradece la colaboración a los miembros del equipo de salud de la Fundación Las Rosas de Ayuda Fraterna, para la realización de este estudio. 